\title{
openheart The heart-gut axis: new target for atherosclerosis and congestive heart failure therapy
}

\author{
Esther Forkosh, Yaron Ilan
}

To cite: Forkosh E, llan Y. The heart-gut axis: new target for atherosclerosis and congestive heart failure therapy. Open Heart 2019;6:e000993. doi:10.1136/ openhrt-2018-000993

Received 12 December 2018 Revised 22 March 2019 Accepted 29 March 2019
Check for updates

\section{(c) Author(s) (or their} employer(s)) 2019. Re-use permitted under CC BY-NC. No commercial re-use. See rights and permissions. Published by BMJ.

Medicine, Hebrew University Hadassah Medical Center, Jerusalem, Israel

Correspondence to Dr Yaron Ilan; ilan@hadassah. org.il

\begin{abstract}
The human gut microbiota has been identified as a possible novel risk factor for cardiovascular disease. The intestinal microbiome plays a role in the pathogenesis of atherosclerosis and heart failure. Even though studies in rodents suggested that gut microbes may affect the risk of heart disease, this link has not been shown in humans. In the present study, we review several potential mechanisms by which the gut microbiome and bacterial translocation are associated with the development of cardiac disorders making them potential targets for novel therapeutic measures for these conditions. Modulation of the gut microbiota as a mechanism for altering the pathogenesis of disorders is an area of growing interest. Alteration in the gut microbiota is being explored as a method of reducing risk factors associated with cardiac diseases.
\end{abstract}

\section{INTRODUCTION}

The intestinal microbiome plays an important role in controlling whole-body metabolic homeostasis and organ physiology. ${ }^{1}$ Cardiovascular diseases (CVD) including coronary heart disease (CHD) are leading causes of mortality in the Western world affecting about one-third of the population. Studies over the last decade suggested a potential role for the gut microbiome in the pathogenesis of atherosclerosis, CVD and heart failure. Even though studies in rodents suggested that gut microbes may affect the risk of heart disease, this link has not been shown in humans. ${ }^{2}$ In the present study, we review several mechanisms for the potential role of the microbiome and bacterial translocation (BT) that may contribute to the pathogenesis of CVD and serve as targets for therapeutic approaches.

\section{THE HEART-GUT AXIS: A ROLE FOR THE GUT MICROBIOME IN THE PATHOGENESIS OF HEART DISEASES \\ Endotoxaemia induces a chronic inflammatory state which contributes to atherosclerosis and CVD}

A persistent low-grade inflammatory response underscores a metabolic syndrome and is also a risk factor for CVD. ${ }^{3}{ }^{4}$ Inflammatory markers are associated with obesity and the risk of obesity-associated CVD. ${ }^{5}$ Perturbation of the intestinal microbiota and changes in gut permeability are triggers for the chronic inflammatory state. ${ }^{5}$ 'Metabolic endotoxaemia' is a term used to describe a link among gut bacteria, endotoxins and their circulating levels, with inflammatory-induced obesity and metabolic diseases linking it to CVD. ${ }^{6}$ The microbiome, with aberrant gut microbiota profiles, is important for the pathogenesis of inflammatory-induced obesity, type 2 diabetes mellitus and other disorders associated with a metabolic syndrome. ${ }^{67}$ Gut microbiota signatures were identified using gut flora analyses in animal obesity, type 1 and type 2 diabetes and non-alcoholic fatty liver disease studies; however, their relevance in humans is yet to be determined. ${ }^{68}$

High serum lipopolysaccharide (LPS) activity is associated with cardiometabolic disorders, which supports the role of bacterial infections and immune responses in their aetiology. ${ }^{9}$ The transfer of microbiota from obese animals induces metabolic disease and obesity in germ-free animals. ${ }^{10}$ Conversely, transfer of pathogen-free microbiota from lean healthy human donors to patients with metabolic disease can increase insulin sensitivity. ${ }^{11-13}$ In a recent study, 2452 patients were followed up for 10 years, and LPS activity was found to be associated with total energy and carbohydrate intake in lean, healthy subjects. High LPS was associated with obesity, metabolic syndrome, diabetes and CHD events, independent of other established risk factors. ${ }^{9}$

\section{Role of the microbiome in the progression of atherosclerosis}

The intestinal microbiota impacts lipid metabolism and may exert a protective effect on atherosclerosis development. ${ }^{14} 15$ Using a low cholesterol diet in the Apoe ${ }^{-/-}$ mouse model, a group raised in germ-free conditions showed a greater development of atherosclerotic plaques than controls did. ${ }^{16}$ 
Treatment of Apoe ${ }^{-/-}$mice with the gut bacteria Akkermansia muciniphila, reduced the size of atherosclerotic plaques, an effect that was attributed to its anti-inflammatory activity. ${ }^{17}$

\section{The interplay between the microbiome and dietary-derived compounds is associated with CVD}

Several dietary-related effects of the gut microbiota contribute to the pathogenesis of CVD. Acute or longterm high-fat diets lead to a rise in endotoxin levels. ${ }^{6}$ Metabolites derived from the gut microbial metabolism of choline, phosphatidylcholine and L-carnitine directly contribute to CVD pathology, which underscores the increased risk of eating too much red meat. ${ }^{8}$ These dietary nutrients have a trimethylamine (TMA) moiety, which participates in the development of atherosclerotic heart disease. ${ }^{18}$ Hepatic production of trimethylamine-N-oxide (TMAO) from gut microbiota-derived TMA enhanced cardiovascular risk. ${ }^{1}$ Levels of both gut microbiota-dependent TMA and hepatic flavin monooxygenase 3-dependent TMAO are predictors of atherosclerosis and CVD, further supporting a link between the gut microbiota and heart disease. ${ }^{18-20}$ In mice, a strong association was noted between atherosclerotic plaque size and plasma TMAO levels. ${ }^{21} \mathrm{~A}$ study of the relationship between fasting plasma choline and betaine levels and the risk of major adverse cardiac events (MACE), which includes death, myocardial infarction and stroke, in relation to TMAO was conducted with 3903 subjects undergoing coronary angiography over 3 years of follow-up. This study showed that higher plasma choline and betaine levels were associated with an increased risk of MACE. ${ }^{19}$ Phosphatidylcholine, TMAO and betaine predicted CVD in an independent large clinical cohort. ${ }^{22}$ TMAO levels correlated with the degree of severity of heart failure and with adverse outcomes. ${ }^{20}$

Gut microbial transplantation can transmit choline diet-induced TMAO production and atherosclerosis susceptibility. ${ }^{21}$ Dietary supplementation of mice with choline and TMAO promoted macrophage scavenger receptors associated with atherosclerosis, while betaine supplementation only promoted macrophage scavenger receptors associated with atherosclerosis. ${ }^{22}$ Suppression of intestinal microflora in atherosclerosis-prone mice inhibited dietary choline-enhanced atherosclerosis. Both phosphatidylcholine/choline and/or L-carnitine are found in large quantities in red meat and were suggested to increase the risk of CVD. Genetic variations controlling the expression of flavin monooxygenases, an enzymatic source of TMAO, segregated with atherosclerosis in hyperlipidaemic mice. ${ }^{22}$ Other studies demonstrated beneficial properties for L-carnitine consumption against metabolic diseases including skeletal muscle insulin resistance and ischaemic heart disease (IHD). Fish is a significant source of TMAO, but dietary fish consumption exerts positive effects on cardiovascular health. ${ }^{1}$

The gut microbiota promotes energy harvest and storage from the diet and is beneficial during periods of nutrient deprivation. ${ }^{23}$ Fasting produces a marked change in gut microbiota, with increased levels of short-chain fatty acids (SCFAs) generated from the microbial fermentation of glycans when compared with germ-free controls. During fasting, a microbiota-dependent, peroxisome proliferator-activated receptor-alpha-regulated increase in hepatic ketogenesis occurs, and myocardial metabolism is directed to ketone body utilisation.

Taken together, these data support a role for the interplay between the gut microbiome and dietary compounds in the pathogenesis of heart disease. ${ }^{23}$

\section{Increased gut permeability as a risk factor for CVD}

An impaired intestinal barrier function is followed by BT, and bacterial products trigger an inflammatory cascade. This has been associated with obesity and insulin resistance. ${ }^{24}$ Moreover, patients with inflammatory bowel diseases (IBDs) who have high permeability of their intestinal barrier suffer from a higher risk of CHD despite a lower prevalence of other risk factors. ${ }^{25}$ The increased long-term risk of IHD in these patients is related to the chronic inflammatory state, and interventions reducing the inflammatory burden may attenuate this risk. ${ }^{26}$ During 1-13 years of follow-up after the diagnosis of IBD, the risk of IHD was high. This risk was lower among patients with IBD using 5-aminosalicylic acids, thiopurines and tumour necrosis factor (TNF) alpha antagonists, or among those treated surgically.

SCFAs are fermented from dietary fibres by the gut microbiota. ${ }^{27}$ The most abundant SCFAs are acetate, propionate and butyrate, which are mostly metabolised in the colon and have numerous effects within the gastrointestinal tract, including maintaining the integrity of the large and small intestinal barrier. SCFAs that reach the systemic circulation were shown to have the ability to modulate CVD risk factors including the reduction of blood pressure and regulation of glucose and lipid homeostasis. ${ }^{28}$

\section{BT is associated with the pathogenesis of heart failure}

BT contributes to congestive heart failure (CHF) leading to a vicious cycle where impaired cardiac function impacts intestinal microcirculation leading to a barrier defect of the intestinal mucosa. ${ }^{25}$ Small intestinal function is altered in decompensated CHF and translocation of LPS contributes to a state of chronic inflammation. ${ }^{29} \mathrm{CHF}$ is associated with a reduction of active and passive carrier-mediated intestinal transport and is more profound in oedematous patients. Active carrier-mediated intestinal transport was reduced in decompensated CHF, indicating epithelial dysfunction due to intestinal ischaemia. Oedematous patients had the highest blood concentrations of LPS, TNF and soluble tumour necrosis factor receptor R1 (sTNF-R1). CHF patients with higher LPS concentrations had the highest concentrations of TNF and sTNF-R1. ${ }^{29}$ 


\section{Composition of gut microbiota in patients with CAD}

Studies comparing the gut microbiota derived from faecal samples of three groups: patients with CAD, healthy volunteers and patients with coronary risk factors without $\mathrm{CAD}$, revealed a significant increase in the order Lactobacillales in the CAD group. In addition, a higher percentage of lactobacilli were found in multivessel diseases than in single-vessel diseases. ${ }^{30}$ A study including almost 12000 participants showed a correlation between poor oral hygiene and CVD events, elevated $\mathrm{C}$ reactive protein and fibrinogen. ${ }^{31}$ The bacteria found in the atherosclerotic plaques predominantly exist in the oral cavity and gut of the same person, suggesting a similar origin indicating the possible contribution of these bacteria to the development of atherosclerosis and CVD. In a study using pyrosequencing of $16 \mathrm{~S}$ rRNA in atherosclerotic plaque, oral, and gut samples of 15 patients with atherosclerosis, a combination of Veillonella sp and Streptococcus sp in atherosclerotic plaques correlated with their abundance in the oral cavity. Chryseomonas sp was identified in all atherosclerotic plaque samples with Veillonella sp and Streptococcus sp identified in a majority of the samples. ${ }^{32}$ Several species, such as Porphyromonas gingivalis and Aggregatibacter actinomycetemcomitans, were shown to cause an increase in plaque size in animal models following an oral or intravenous infection. ${ }^{33-35}$

\section{An interplay between the gut microbiome with gut and systemic hormones affects CVD}

Gut microbiome alterations are related to changes in gut hormones. ${ }^{36}$ Decreased intestinal signalling for fats was described in mice lacking gut microbiota. ${ }^{37}$ Plasma levels of the energy homeostasis hormones, ghrelin and PYY 3-36, are associated with left ventricular mass indices. These associations indicate a possible interaction between gut peptides and the cardiovascular system in hypertension and obesity. ${ }^{38}$

The gut microbiota affects different tissues, adipose deposits, hormonal, pharmacological, nutritional and life style factors, and can also affect adiponectin clearance and release from T-cadherin-associated tissue reservoirs. ${ }^{39}$ Altered adiponectin levels are present in patients with heart failure. Inflammation downregulates adiponectin production and its levels are reduced in obesity and its associated comorbidities. ${ }^{39}$ A positive association between inflammation and adiponectin has been reported in inflammatory disorders, in contrast with the negative correlation typical of metabolic diseases.

\section{The interplay of the gut microbiome with bile acid metabolites affects the pathogenesis of heart disorders}

Bile acids are associated with signalling. Gut microbial depletion affects the bile acid submetabolome of several organs including the heart in rats. ${ }^{40}$ Unconjugated bile acids comprise the largest proportion of the total measured bile acid profile in the heart. In contrast, taurine-conjugated bile acids (taurocholic acid and tauro-beta-muricholic acid) dominate the cardiac bile acid profile in germ-free animals. These communication networks are affected by microbial activities noted by farnesoid $\mathrm{X}$ receptor-regulated pathway transcripts. The presence of specific microbial bile acid cometabolite patterns in the heart suggests a signalling role for these compounds and highlights the extent of gut microbiome effects on these pathways. ${ }^{40}$

\section{TARGETING THE GUT MICROBIOME AS A TREATMENT FOR CVD}

The intestinal microbiome represents a new potential therapeutic target for the treatment of cardiometabolic diseases. ${ }^{41}$ Intervention studies in humans aiming to selectively alter the composition of the microbiota or to pharmacologically manipulate the microbiota to influence production of their metabolites are being explored. Several examples for such manipulations are described below.

\section{Probiotics}

Probiotics are live microorganisms which are beneficial to the host. ${ }^{42}$ Human studies with probiotic strains show that ingestion of viable microorganisms with an ability to hydrolyse bile salts lowers blood cholesterol, alleviating a risk factor for CVD. ${ }^{8}$ Certain probiotic bacterial strains reduce cholesterol and hypertension. ${ }^{42-46}$

Studies in animals suggested that probiotics can attenuate heart failure. ${ }^{47}$ Oral probiotic Lactobacillus rhamnosus GR-1 administration altered the progression of postinfarction heart failure. Animals-administered GR-1 exhibited a significant attenuation of left ventricular hypertrophy, and improved systolic and diastolic left ventricular function. Metabolomic analysis showed differences in the preservation of myocardial taurine levels in treated mice. Beneficial effects were also evident after the cessation of therapy, suggesting the persistence of the GR-1 effect. ${ }^{47}$

\section{Prebiotics}

Increased consumption of whole-plant foods, including fruits, vegetables and whole-grain cereals, provides the rational for efficacious prebiotics. Prebiotics modulate the gut microbiota exerting an inverse correlation with the risk of cardiometabolic diseases. ${ }^{48}$ Diets based on the high intake of fermentable fibres and plant polyphenols alter the microbial activities within the gut. ${ }^{8}$ In a study by Marques et al, a high-fibre diet and acetate supplementation reduced blood pressures, cardiac fibroses and left ventricular hypertrophies. ${ }^{49}$ Fibres and polyphenols are converted into biologically active compounds by the colonic microbiome thereby upregulating the colon-systemic metabolic axis. ${ }^{50}$ Polyphenols derived from dietary plants exert a protective effect on vascular endothelial cells using antioxidants that prevent the oxidation of low-density lipoprotein. ${ }^{51}$

Cranberry Vaccinium macrocarpon Aiton extract (CE) reduced the high fat/high sucrose-induced weight gain, visceral obesity, liver weight and triglyceride accumulation in mice. ${ }^{52} \mathrm{CE}$ administration improved insulin sensitivity, lowered intestinal triglyceride content and 
alleviated intestinal inflammation and oxidative stress. $\mathrm{CE}$ treatment increased the proportion of the mucin-degrading bacterium Akkermansia sp in the metagenomic samples.

A positive association between increased dietary intake of whole grains and a reduced risk of cardiometabolic disorders has been shown. ${ }^{48}$ Wholegrain foods have been associated with lower blood glucose. In vitro, total bacterial populations increased significantly in cultures when cereal samples were supplemented with $\mathrm{pH}$-controlled anaerobic cultures of human faecal microbiota. Proliferation of the genus Bifidobacterium and Lactobacillus-Enterococcus groups were noted. ${ }^{48}$

\section{Dietary measures that alter the immune system of the gut}

Selection of health promoting foods and the design of functional foods are being explored as a means of promoting a 'healthy' microbiome. ${ }^{50}$ Circulating LPS arising from gastrointestinal tract microbiota is associated with both infection and inflammation, and may be affected by daily nutrition. ${ }^{9}$ Strategies that modulate the gut microbiota or their metabolic activities by wholeplant foods, probiotics and prebiotics may be at the base of healthy eating pyramids advised by regulatory agencies and can decrease the risk for CVD. ${ }^{8}$

Consumption of a Western diet, which is low in dietary fibre and fermentable substrates and high in saturated and transfatty acids, is associated with the depletion of metabolic fuels, resulting in an alteration of the gut microbial profile and contributes to increased cholesterol levels. ${ }^{53}$ End products of bacterial fermentation, particularly the SCFAs (propionate), may be associated with this increase. A shift towards a plant-based diet may confer protective effects against atherosclerotic CAD by increasing endothelial protective factors in the circulation while reducing factors that are injurious to endothelial cells. ${ }^{51}$

\section{Targeting the bacterial LPS}

In the Bruneck study, plasma levels of bacterial LPS constitute a strong risk factor for CVD. LPS levels were measured in a random population of 516 subjects aged 50-79 years. Subjects with elevated serum levels beyond the 90th percentile faced a threefold increased risk of incident atherosclerosis, which is more pronounced in subjects with chronic infections and in current and ex-smokers. ${ }^{54-57}$

Interventions that target 'leaky' mucosal membranes, endotoxin-coupled lipid absorption or removal of circulating endotoxins, can reduce the progression of inflammatory-induced metabolic diseases. ${ }^{6}$ Bovine colostrums were shown to improve gut BT. In vitro, this reduced apoptosis is measured by active caspase- 3 and caspase- 9 , baxalpha, Bcl-2, heat shock protein 70 expression and epithelial electrical resistance in colonic cell lines. ${ }^{58} \mathrm{In}$ both animals and humans, oral administration of bovine colostrum-derived anti-LPS compounds were associated with the alleviation of insulin resistance, hyperlipidaemia and liver damage associated with the metabolic syndrome. ${ }^{59-61}$

Lactoferrin (LF) is a natural immunomodulator that regulates immune responses including the control of inflammatory cytokine production during acute inflammation. ${ }^{62}$ LF administered prior to LPS protected rats from LPS-induced hypotension. The effect was associated with a decrease in serum TNF-alpha and interleukin 6 , and with histological protection of intestinal tissue post LPS administration. ${ }^{62}$

\section{Bariatric surgery as a means for altering gut microbiome}

Bariatric procedures such as the Roux-en-Y gastric bypass (RYGB) operation improve obesity-associated metabolic disorders in addition to their weight loss effects. In a rat model, plasma bile acids, phosphocholines, amino acids, energy-related metabolites, nucleoside and amine metabolites, cardiac glycogen and amino acids were altered following the RYGB procedure. These surgically induced metabolic shifts were associated with an alteration of the gut microbiota. ${ }^{63}$ These changes reflected an enhancement of cardiac energy metabolism through tricarboxylic acid cycle intermediates, cardiorenal protective activities and biochemical caloric restrictions.

\section{Physical activity as a means of altering the gut microbiome}

Exercise can also alter the gut microbiota. Exercise increases faecal concentrations of SCFA in lean participants compared with obese ones. ${ }^{64}$ In a mouse model, transplanting the gut microbiome from mice that received exercise to germ-free mice led to weight gain and to a significant elevation of the butyrate:acetate ratio. ${ }^{64}$ This finding supports the assumption that gut production of SCFAs helps to enhance energy within a physically active mouse, thereby becoming a survival benefit during caloric deficient periods. ${ }^{65}$

\section{The microbiome-drug interaction}

The intestinal microbiome plays a role in drug bioavailability, activity and toxicity. ${ }^{66}$ The high variability in the bioavailability of digoxin among individuals was suggested to be associated with alterations in the microbiome. Inactivation of digoxin was found when it was incubated in vitro with the gut bacterium Eggerthella lenta. Arginine supplements might be a potential intervention in increasing digoxin activity by inhibiting the expression of cardiac glycoside reductase gene operons that can inactivate digoxin. ${ }^{66}$

\section{SUMMARY}

The gut microbiome and BT are associated with the development of CVD. Modulation of the gut microbiota is an area of growing interest as a means of altering the pathogenesis and complications of cardiac disorders. Altering the gut microbiota may become an attractive method for reducing risk factors and minimising the severity and complications associated with these disorders. 
Contributors Both authors wrote the manuscript.

Funding The authors have not declared a specific grant for this research from any funding agency in the public, commercial or not-for-profit sectors.

Competing interests None declared.

Patient consent for publication Not required.

Provenance and peer review Not commissioned; externally peer reviewed.

Data availability statement There are no data in this work.

Open access This is an open access article distributed in accordance with the Creative Commons Attribution Non Commercial (CC BY-NC 4.0) license, which permits others to distribute, remix, adapt, build upon this work non-commercially, and license their derivative works on different terms, provided the original work is properly cited, appropriate credit is given, any changes made indicated, and the use is non-commercial. See: http://creativecommons.org/licenses/by-nc/4.0/.

\section{REFERENCES}

1. Ussher JR, Lopaschuk GD, Arduini A. Gut microbiota metabolism of L-carnitine and cardiovascular risk. Atherosclerosis 2013;231:456-61.

2. Gut microbes may affect heart disease risk. but studies in rodents suggesting a link may not play out in people. Harv Heart Lett 2012;22.

3. Hotamisligil GS. Inflammation and metabolic disorders. Nature 2006;444:860-7.

4. Hotamisligil GS. Endoplasmic reticulum stress and the inflammatory basis of metabolic disease. Cell 2010;140:900-17.

5. Cox AJ, West NP, Cripps AW. Obesity, inflammation, and the gut microbiota. Lancet Diabetes Endocrinol, 2014.

6. Piya MK, Harte AL, McTernan PG. Metabolic endotoxaemia: is it more than just a gut feeling? Curr Opin Lipidol 2013;24:78-85.

7. Ilan Y. Immune therapy for nonalcoholic steatohepatitis: are we there yet? J Clin Gastroenterol 2013;47:298-307.

8. Tuohy KM, Fava F, Viola R. 'The way to a man's heart is through his gut microbiota' - dietary pro- and prebiotics for the management of cardiovascular risk. Proc. Nutr. Soc. 2014;73:172-85.

9. Kallio KAE, Hätönen KA, Lehto M, et al. Endotoxemia, nutrition, and cardiometabolic disorders. Acta Diabetol 2015;52.

10. Henao-Mejia J, Elinav E, Jin C, et al. Inflammasome-mediated dysbiosis regulates progression of NAFLD and obesity. Nature 2012;482:179-85.

11. Xu M-Q, Cao H-L, Wang W-Q, et al. Fecal microbiota transplantation broadening its application beyond intestinal disorders. World $\mathrm{J}$ Gastroenterol 2015;21:102-11.

12. Han J-L, Lin H-L. Intestinal microbiota and type 2 diabetes: from mechanism insights to therapeutic perspective. WJG 2014;20:17737-45.

13. Smits LP, Bouter KEC, de Vos WM, et al. Therapeutic potential of fecal microbiota transplantation. Gastroenterology 2013;145:946-53.

14. Chistiakov DA, Bobryshev YV, Kozarov E, et al. Role of gut microbiota in the modulation of atherosclerosis-associated immune response. Front Microbiol 2015;6.

15. Battson ML, Lee DM, Weir TL, et al. The gut microbiota as a novel regulator of cardiovascular function and disease. J Nutr Biochem 2018;56:1-15

16. Stepankova R, Tonar Z, Bartova J, et al. Absence of microbiota (germ-free conditions) accelerates the atherosclerosis in apoE-deficient mice fed standard low cholesterol diet. JAT 2010;17:796-804.

17. Li J, Lin S, Vanhoutte PM, et al. Akkermansia Muciniphila Protects Against Atherosclerosis by Preventing Metabolic Endotoxemia-Induced Inflammation in Apoe ${ }^{-1-}$ Mice. Circulation 2016;133:2434-46.

18. Tang WHW, Hazen SL. The contributory role of gut microbiota in cardiovascular disease. J. Clin. Invest. 2014;124:4204-11.

19. Wang Z, Tang WHW, Buffa JA, et al. Prognostic value of choline and betaine depends on intestinal microbiota-generated metabolite trimethylamine-N-oxide. European Heart Journal 2014;35:904-10.

20. Trøseid M, Ueland T, Hov JR, et al. Microbiota-dependent metabolite trimethylamine- $\mathrm{N}$-oxide is associated with disease severity and survival of patients with chronic heart failure. J Intern Med 2015;277.

21. Gregory JC, Buffa JA, Org E, et al. Transmission of atherosclerosis susceptibility with gut microbial transplantation. J Biol Chem 2015;290:5647-60.

22. Wang Z, Klipfell E, Bennett BJ, et al. Gut flora metabolism of phosphatidylcholine promotes cardiovascular disease. Nature 2011;472:57-63.
23. Sim K, Shaw AG, Randell P, et al. Dysbiosis Anticipating necrotizing enterocolitis in very premature infants. Clin Infect Dis 2015;60.

24. Araújo JR, Tomas J, Brenner C, et al. Impact of high-fat diet on the intestinal microbiota and small intestinal physiology before and after the onset of obesity. Biochimie 2017;141:97-106.

25. Rogler G, Rosano G. The heart and the gut. Eur Heart $J$ 2014;35:426-30.

26. Rungoe $\mathrm{C}$, Basit S, Ranthe MF, et al. Risk of ischaemic heart disease in patients with inflammatory bowel disease: a nationwide Danish cohort study. Gut 2013;62:689-94.

27. Chambers ES, Preston T, Frost G, et al. Role of gut MicrobiotaGenerated short-chain fatty acids in metabolic and cardiovascular health. Curr Nutr Rep 2018;7:198-206.

28. Edwards CA, Havlik J, Cong W, et al. Polyphenols and health: interactions between fibre, plant polyphenols and the gut microbiota. Nutr Bull 2017;42:356-60.

29. Sandek A, Bjarnason I, Volk H-D, et al. Studies on bacterial endotoxin and intestinal absorption function in patients with chronic heart failure. International Journal of Cardiology 2012;157:80-5.

30. Emoto T, Yamashita T, Kobayashi T, et al. Characterization of gut microbiota profiles in coronary artery disease patients using data mining analysis of terminal restriction fragment length polymorphism: gut microbiota could be a diagnostic marker of coronary artery disease. Heart Vessels 2017;32:39-46.

31. de Oliveira C, Watt R, Hamer M. Toothbrushing, inflammation, and risk of cardiovascular disease: results from Scottish health survey. BMJ 2010;340:c2451.

32. Koren O, Spor A, Felin J, et al. Human oral, gut, and plaque microbiota in patients with atherosclerosis. Proceedings of the National Academy of Sciences 2011;108(Supplement_1):4592-8.

33. Kramer CD, Simas AM, He X, et al. Distinct roles for dietary lipids and Porphyromonas gingivalis infection on atherosclerosis progression and the gut microbiota. Anaerobe 2017;45:19-30.

34. Liljestrand JM, Paju S, Pietiäinen M, et al. Immunologic burden links periodontitis to acute coronary syndrome. Atherosclerosis 2018;268:177-84.

35. Atarbashi-Moghadam F, Havaei SR, Havaei SA, et al. Periopathogens in atherosclerotic plaques of patients with both cardiovascular disease and chronic periodontitis. ARYA Atheroscler 2018;14:53-7.

36. Cluny NL, Eller LK, Keenan CM, et al. Interactive effects of oligofructose and obesity predisposition on gut hormones and microbiota in diet-induced obese rats. Obesity 2015;23:769-78.

37. Duca FA, Swartz TD, Sakar Y, et al. Increased oral detection, but decreased intestinal signaling for fats in mice lacking gut microbiota. PLoS One 2012;7:e39748.

38. Karcz-Socha I, Zwirska-Korczala K, Zembala M, et al. Ghrelin PYY 3-36 serum changes in left ventricular hypertrophic, insulin-resistant, hypertensive obese patients. Obes Facts 2011;4:386-92.

39. Fantuzzi G. Adiponectin in inflammatory and immune-mediated diseases. Cytokine 2013;64:1-10.

40. Swann JR, Want EJ, Geier FM, et al. Systemic gut microbial modulation of bile acid metabolism in host tissue compartments. Proceedings of the National Academy of Sciences 2011;108(Supplement_1):4523-30.

41. Vinjé S, Stroes E, Nieuwdorp M, et al. The gut microbiome as novel cardio-metabolic target: the time has come! Eur Heart $J$ 2014;35:883-7.

42. Ettinger G, MacDonald K, Reid G, et al. The influence of the human microbiome and probiotics on cardiovascular health. Gut Microbes 2014;5:719-28.

43. Kassaian N, Feizi A, Aminorroaya A, et al. Probiotic and synbiotic supplementation could improve metabolic syndrome in prediabetic adults: a randomized controlled trial. Diabetes \& Metabolic Syndrome: Clinical Research \& Reviews 2018.

44. He M, Shi B. Gut microbiota as a potential target of metabolic syndrome: the role of probiotics and prebiotics. Cell Biosci 2017;7.

45. Cicero AFG, Colletti A, Bajraktari G, et al. Lipid-lowering nutraceuticals in clinical practice: position paper from an international lipid expert panel. Nutr Rev 2017;75:731-67.

46. Hendijani F, Akbari V. Probiotic supplementation for management of cardiovascular risk factors in adults with type II diabetes: a systematic review and meta-analysis. Clinical Nutrition 2018;37:532-41.

47. Gan XT, Ettinger G, Huang CX, et al. Probiotic administration attenuates myocardial hypertrophy and heart failure after myocardial infarction in the rat. Circ Heart Fail 2014;7:491-9.

48. Connolly ML, Tuohy KM, Lovegrove JA. Wholegrain oat-based cereals have prebiotic potential and low glycaemic index. $\mathrm{Br} J$ Nutr 2012;108:2198-206. 
49. Marques FZ, Nelson E, Chu P-Y, et al. High-fiber diet and acetate supplementation change the gut microbiota and prevent the development of hypertension and heart failure in hypertensive mice. Circulation 2017:135:964-77.

50. Fava F, Lovegrove JA, Gitau R, et al. The gut microbiota and lipid metabolism: implications for human health and coronary heart disease. Curr Med Chem 2006;13:3005-21.

51. Tuso P, Stoll SR, Li WW, et al. A plant-based diet, atherogenesis, and coronary artery disease prevention. Perm J 2015;19:62-7.

52. Anhê FF, Roy D, Pilon G, et al. A polyphenol-rich cranberry extract protects from diet-induced obesity, insulin resistance and intestinal inflammation in association with increased Akkermansia spp. population in the gut microbiota of mice. Gut 2015;64.

53. Wong JMW, Esfahani A, Singh N, et al. Gut microbiota, diet, and heart disease. J AOAC Int 2012;95:24-30.

54. Mayr M, Kiechl S, Tsimikas S, et al. Oxidized low-density lipoprotein autoantibodies, chronic infections, and carotid atherosclerosis in a population-based study. Journal of the American College of Cardiology 2006;47:2436-43.

55. Kiechl S, Willeit J. In a nutshell: findings from the Bruneck study. Gerontology 2019;65:9-19.

56. Wiedermann CJ, Kiechl S, Dunzendorfer S, et al. Association of endotoxemia with carotid atherosclerosis and cardiovascular disease: prospective results from the Bruneck study. J Am Coll Cardiol 1999;34:1975-81.

57. Wiedermann CJ, Kiechl S, Schratzberger P, et al. The role of immune activation in endotoxin-induced atherogenesis. Journal of Endotoxin Research 2001;7:322-6.

58. Marchbank T, Davison G, Oakes JR, et al. The nutriceutical bovine colostrum truncates the increase in gut permeability caused by heavy exercise in athletes. Am J Physiol Gastrointest Liver Physiol 2011;300:G477-G484.

59. Mizrahi M, Shabat Y, Ben Ya'acov A, et al. Alleviation of insulin resistance and liver damage by oral administration of Imm124-E is mediated by increased Tregs and associated with increased serum GLP-1 and adiponectin: results of a phase I/II clinical trial in NASH. $J$ Inflamm Res 2012;5:141-50.

60. Ilan Y. Leaky gut and the liver: a role for bacterial translocation in nonalcoholic steatohepatitis. WJG 2012;18:2609-18.

61. Adar T, Ben Ya'acov A, Lalazar G, et al. Oral administration of immunoglobulin G-enhanced colostrum alleviates insulin resistance and liver injury and is associated with alterations in natural killer T cells. Clin Exp Immunol 2012;167:252-60.

62. Doursout M-F, Horton H, Hoang L, et al. Lactoferrin moderates LPSinduced hypotensive response and gut injury in rats. International Immunopharmacology 2013;15:227-31.

63. Ashrafian H, Li JV, Spagou K, et al. Bariatric surgery modulates circulating and cardiac metabolites. J. Proteome Res. 2014;13:570-80.

64. Allen JM, Mailing LJ, Cohrs J, et al. Exercise training-induced modification of the gut microbiota persists after microbiota colonization and attenuates the response to chemically-induced colitis in gnotobiotic mice. Gut Microbes 2018:9:115-30.

65. LeBlanc JG, Chain F, Martín R, et al. Beneficial effects on host energy metabolism of short-chain fatty acids and vitamins produced by commensal and probiotic bacteria. Microb Cell Fact 2017;16.

66. Lu L, Wu Y, Zuo L, et al. Intestinal microbiome and digoxin inactivation: meal plan for digoxin users? World J Microbiol Biotechnol 2014;30:791-9. 